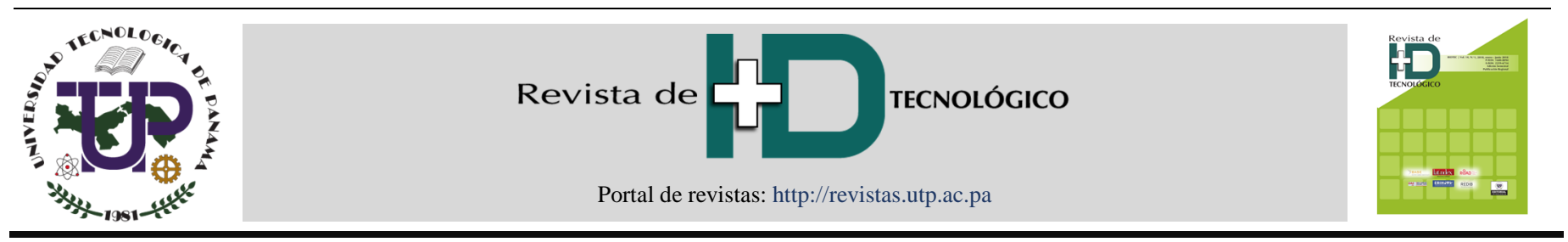

\title{
Estudio experimental del concreto poroso con la incorporación de distintas
} granulometrías

\section{Experimental study of porous concrete with the incorporation of different granulometries}

\author{
Manuel R. Pinto ${ }^{1 *}$, Clara I. Carrasco ${ }^{2}$, Karen E. Caballero ${ }^{3}$ \\ 1,2,3 Grupo de Investigación: Ciencia y Tecnología del Concreto (CITEC), Universidad Tecnológica de Panamá, Panamá \\ *Autor de correspondencia: marodrigo11@hotmail.com
}

\begin{abstract}
RESUMEN- El concreto poroso por lo general es un tipo de concreto sin agregado fino cuya principal característica y diferencia del concreto convencional es su permeabilidad, gracias a su estructura porosa. En este documento se presenta un estudio del comportamiento del concreto poroso en su resistencia a compresión y permeabilidad con diferentes nominaciones de grava, con la finalidad de encontrar resistencias que vayan acuerdo a las diferentes aplicaciones en campo como aceras y hombros de pavimentos. Para lograrlo se utilizaron diferentes granulometrías con diámetros de grava que van de 3/4" a 3/8", cemento tipo 1 estructural y aditivos. Como resultado se obtuvo las propiedades en estado fresco como el revenimiento y propiedades en estado endurecido como la permeabilidad y las resistencias a compresión de cada serie presentada y su variación a medida que se cambia el tipo de agregado.
\end{abstract}

Palabras clave - concreto poroso, permeabilidad, porosidad, tasa de filtración.

\begin{abstract}
Porous concrete is usually a type of concrete without fine aggregate whose main characteristic and difference from conventional concrete is its permeability, thanks to its porous structure. This paper presents a study of the behavior of porous concrete in its resistance to compression and permeability with different gravel nominations, in order to find resistances that are suitable for different applications in the field such as sidewalks and pavement shoulders. To achieve this, different granulometries with gravel diameters ranging from $3 / 4$ "to $3 / 8$ ", structural type 1 cement and additives were used, resulting in properties in the fresh state such as slump and hardened properties such as permeability. and the compression resistance of each series presented and its variation as the type of aggregate is changed.
\end{abstract}

Keywords-porous concrete, permeability, porosity, filtration rate.

\section{Introducción}

El hormigón poroso es un hormigón sin finos, o con pocos finos, con una relación agua/cemento baja, que se usa principalmente en aplicaciones de poco volumen. Es un hormigón de baja resistencia, seco, permeable, que se puede usar para crear un pavimento de buena calidad estructural, que drene el agua de lluvia, lo que reduce el escurrimiento y favorece el abastecimiento de las aguas subterráneas [1].

El diseño de concreto poroso en pavimentos de estructura porosa, en obras de vías y carreteras, proporciona los siguientes beneficios: disminuye el riesgo en la circulación en presencia de lluvia, disminuye los gradientes térmicos y de humedad, y el material presta dos funciones adicionales: drenaje y auto ventilación; al aumentar la estructura de los macro poros. Esto como consecuencia de la infiltración del agua en la superficie del pavimento [2].

Existen diferentes estudios y aplicaciones que se le ha realizado al concreto poroso, Orozco [3] realizó estudios sobre la porosidad del concreto y los efectos que tiene en la durabilidad y la resistencia.

Por otro lado, Vladimir et. al [4] realizaron estudios sobre el comportamiento del concreto poroso bajo un agregado grueso específico con diferentes porcentajes de vacíos en su diseño, de esta manera obteniendo una comparativa entre la resistencia obtenida con el porcentaje de vacíos.

En cuanto a la diferencia que hay entre un concreto convencional y el concreto poroso, los autores Tennis et. al [5] mencionan que, en los pavimentos permeables, aparte de tomar en cuenta para su diseño la resistencia a la carga aplicada y sus propiedades mecánicas, como en

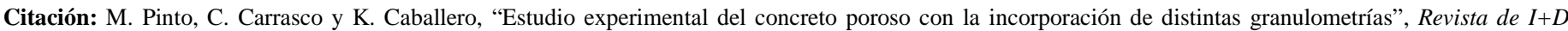
Tecnológico, vol. 14, no. 2, pp. (57-65), 2018.

Tipo de artículo: Original. Recibido: 1 de marzo de 2018. Recibido con correcciones: 20 de septiembre de 2018 . Aceptado: 16 de octubre de 2018 .

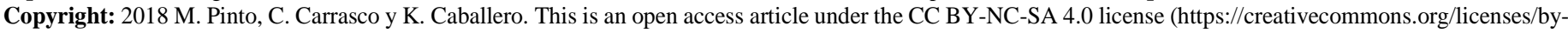
nc-sa/4.0/). 
los pavimentos convencionales, también se toman en cuenta factores hidráulicos como la relación de vacíos y permeabilidad.

El objetivo principal de este trabajo es evaluar el comportamiento del concreto poroso con la incorporación de diferentes granulometrías y de esta manera obtener resistencias adecuadas para la aplicación en campo.

\section{Programa experimental}

Para la presente investigación se utilizó los 3 tipos de grava como especifica la Norma ACI 522R-06 [6], las cuales van de 3/4" como tamaño máximo hasta 3/8" como tamaño de agregado mínimo. En el estudio se eliminó el uso de agregado fino, arena.

Para llevar un orden de los resultados se propuso seis diferentes tipos de series para evaluar las propiedades con agregados de una sola nominación y también combinados, las cuales se muestran a continuación en la tabla 1 .

Tabla 1. Series de concreto poroso propuestos

\begin{tabular}{|c|l|}
\hline $\begin{array}{c}\text { Número de } \\
\text { Serie }\end{array}$ & \multicolumn{1}{|c|}{$\begin{array}{c}\text { Concreto poroso } \\
\text { (Nominación de grava) }\end{array}$} \\
\hline Serie 1 & Agregado 3/4" \\
\hline Serie 2 & Agregado 1/2" \\
\hline Serie 3 & Agregado 3/8" \\
\hline Serie 4 & $\begin{array}{l}\text { Agregado combinado 75\% de 3/4" y } \\
25 \% \text { de 3/8" }\end{array}$ \\
\hline Serie 5 & $\begin{array}{l}\text { Agregado combinado 75\% de } 1 / 2 " \text { y } \\
25 \% \text { de 3/8” }\end{array}$ \\
\hline Serie 6 & Agregado premezclado \\
\hline
\end{tabular}

En el diseño de mezcla del concreto poroso es de suma importancia garantizar resistencias que sean factibles para diferentes aplicaciones en la rama de la ingeniería civil, así como también su permeabilidad.

Estas resistencias son alcanzadas mediante parámetros que definen el comportamiento de este tipo de concreto, los cuales se mencionan a continuación.

\subsection{Relación agua/ cemento}

La relación agua/cemento en el concreto poroso es sumamente importante, ya que modifica las resistencias e influyen en la estructura de vacíos. Según la Norma ACI 522R-06 [6], se usan generalmente relaciones agua/cemento que van desde el rango de 0.26 a 0.45 , dependiendo del agregado y el uso de aditivos. En nuestra investigación se utilizó una relación agua/cemento de 0.36 , ya que por estudios experimentales previos, utilizando relaciones agua/cemento de $0.57,0.45,0.40 \mathrm{y}$ 0.38 , la pasta de cemento se escurría al fondo y no contribuía a la cohesión y adherencia de la grava dentro de la mezcla, tapando también los poros, afectando su permeabilidad. Mientras que utilizando una relación agua/cemento de 0.33 y 0.30 la pasta de cemento quedaba parcialmente seca por la poca cantidad de agua que contenía. A continuación, en las figuras 1,2 y 3 se muestran los efectos de las relaciones agua/cemento dentro de la matriz del concreto.

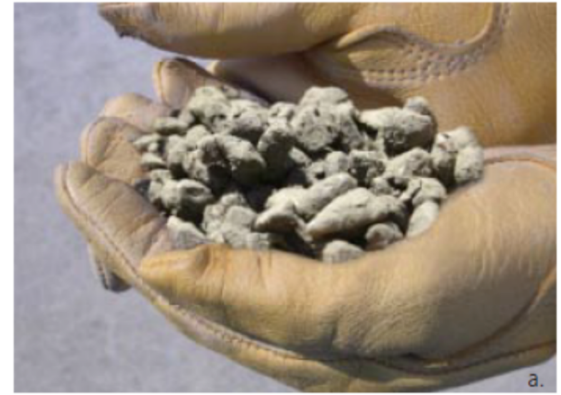

Figura 1. Muestra de concreto poroso con poca cantidad de agua. Tennis et. al [5]

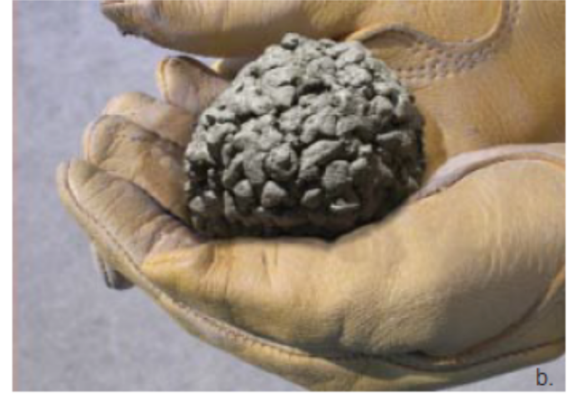

Figura 2. Muestra de concreto poroso con adecuada cantidad de agua. Tennis et. al [5].

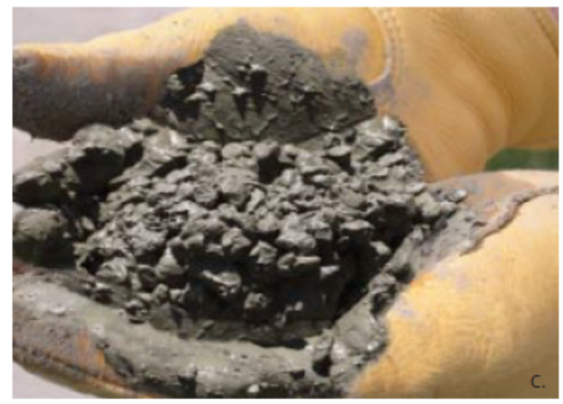

Figura 3. Muestra de concreto poroso con mucha cantidad de agua. Tennis et. al [5]. 


\subsection{Relación agregado/ cemento}

Se utilizó una relación de agregado/cemento de 1:4 según el criterio de Tennis et. al [5] para el agregado de $3 / 4$ ", es decir 1 parte de cemento y 4 partes de agregado, ya que este agregado al ser de mayor tamaño necesitaba más pasta para cubrir las partículas de este agregado y así obtener mejor cohesión entre las partículas. Mientras que para los otros agregados 1/2" y 3/8" se utilizó una relación de agregado/cemento de 1:4.5 según Tennis et. al [5], es decir 1 parte de cemento y 4.5 partes de agregado.

\subsection{Revenimiento}

La prueba de revenimiento o como también se le conoce, prueba de asentamiento, es el ensayo que se le realiza al concreto para medir la consistencia de la misma. Esta prueba se realizó según la norma ASTM C143 [7] la cual es la que se utiliza para concreto convencional.

\subsection{Resistencia a compresión}

El ensayo de resistencia a compresión se regula bajo la norma ASTM C39 [8], la cual presenta los diferentes parámetros y procedimientos a seguir para realizar la prueba, así como también el equipo a utilizar.

Todos los cilindros ensayados se les confeccionó una capa de yeso y cemento en ambas superficies, esto para garantizar una superficie plana y evitar errores en el ensayo. La base del cilindro y la superficie donde iba ser aplicada la carga, eran rugosas y no uniformes, debido al tamaño de las partículas. Gracias a la superficie de yeso y cemento, el pistón de la máquina de compresión bajaba totalmente centrado y uniforme en todo el cilindro, evitando de esta manera errores en los resultados. Los más comunes varían entre $140 \mathrm{~kg} / \mathrm{cm}^{2}$ y $176 \mathrm{~kg} / \mathrm{cm}^{2}$ según la Norma ACI 522R-06 [6].

A continuación, en la figura 4 se muestran los cilindros sin la superficie de yeso y cemento y en la figura 5 se muestra los cilindros con la superficie ya descrita.

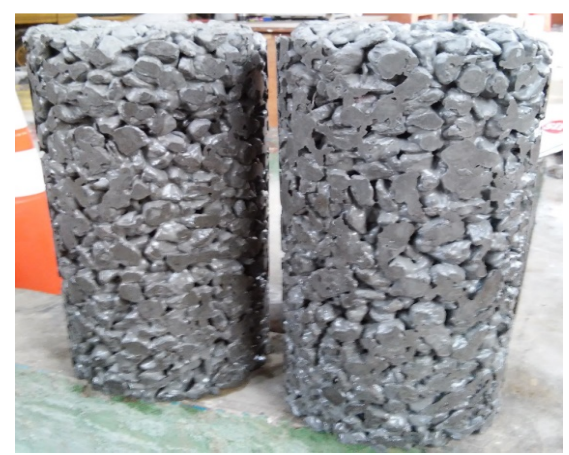

Figura 4. Cilindros de concreto poroso sin superficie de yeso y cemento.

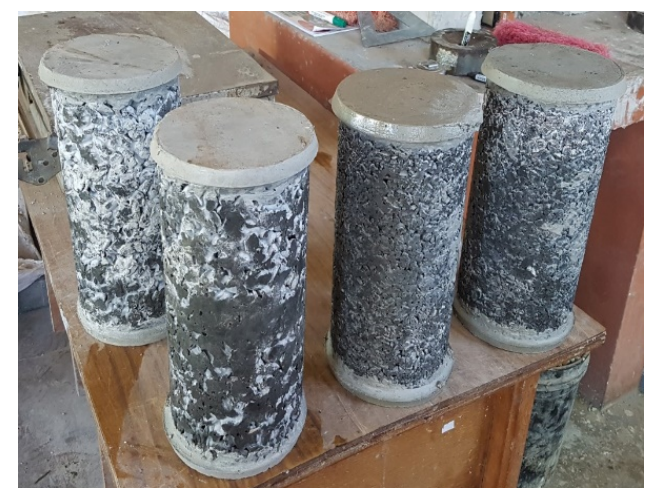

Figura 5. Cilindros de concreto poroso con superficie de yeso y cemento.

\subsection{Permeabilidad}

El ensayo de permeabilidad busca determinar la tasa de filtración que se tiene en el concreto poroso. Para realizar la prueba de permeabilidad se confeccionó una losa con dimensiones ya descritas anteriormente de 16 " largo x 16" de ancho x 3" de altura, la cual fue probada para ver su capacidad de filtración.

La prueba de permeabilidad se realizó según los parámetros descritos en la Norma ASTM C1701 [9]. Consiste en verter agua en una losa de concreto poroso previamente fabricada y cronometrar el tiempo, de esta manera calcular la tasa de filtración. El lugar donde se vaya a realizar la prueba no debe tener ningún tipo de inclinación y debe estar limpio.

Se debe colocar un anillo de filtración de 11.5" a 12" de diámetro, de esta manera controlar el espacio donde se va a verter.

A continuación, en la figura 6 se muestra la instalación del anillo de filtración en la losa de concreto, al igual que el sistema completo para realzar la prueba. 


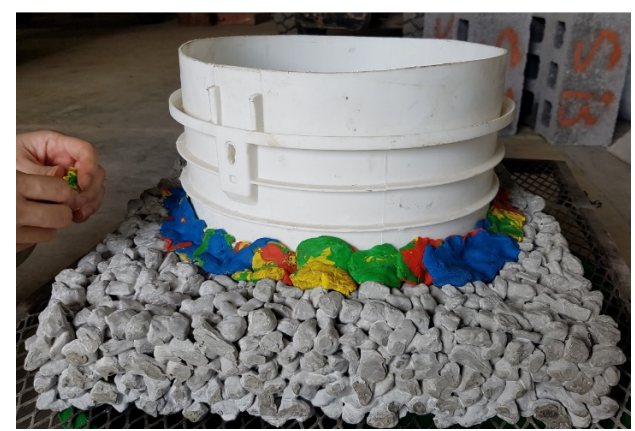

Figura 6. Anillo de filtración de 11.5" de diámetro.

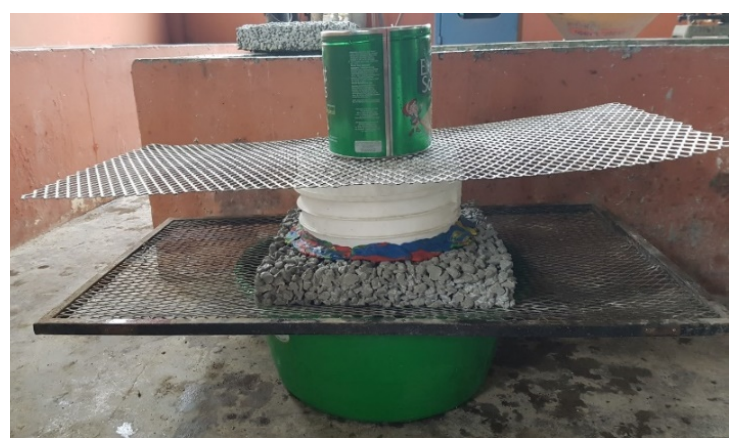

Figura 7. Sistema de captación de agua completo para prueba de filtración.

El agua fue filtrada por la losa, mientras esto ocurría se estará cronometrando el tiempo. El tiempo se cronometra desde que el agua toca la superficie del concreto poroso hasta que este deje de filtrar.

Una vez cronometrado el tiempo se procede a realizar los cálculos según la Norma ASTM C1701 [9].

Para calcular la tasa de infiltración (I) utilizando unidades consistentes se utiliza la siguiente fórmula que describe la norma Norma ASTM C1701 [9].

$$
I=\frac{K M}{\left(D^{2} x t\right)}
$$

En donde:

$\mathrm{I}=$ Tasa de infiltración, $\mathrm{mm} / \mathrm{h}$ (pulgadas/horas)

$\mathrm{M}=$ Masa de infiltración de agua, $\mathrm{kg}$ (lb)

$\mathrm{D}=$ Diámetro interior del anillo de infiltración, $\mathrm{mm}$ (pulg)

$\mathrm{t}=$ Tiempo requerido para que una cantidad medida de agua se infiltre en el concreto, (s)

$\mathrm{K}=4,583,666,000\left(\mathrm{~mm}^{3} \mathrm{x} \mathrm{seg}\right) /(\mathrm{kg} /$ hora $)$ en el Sistema Internacional o 126,870 ( pulg $\left.^{3} \mathrm{seg}\right) /$ (lb x hora) en el Sistema Pulgada-Libra.
Los valores más comunes varían entre $18 \mathrm{gal} / \mathrm{min} / \mathrm{pies}^{2}$ (81 to $730 \mathrm{~L} / \mathrm{min} / \mathrm{m}^{2}$ ) según la Norma ACI 522R-06 [6].

\section{Diseño de mezcla de concreto poroso}

El concreto poroso al no tener agregado fino se dificulta su manejabilidad, ya que este comienza a fraguar rápidamente. Para mejorar este efecto se utilizó un aditivo superplastificante-retardante el cual mejoró la trabajabilidad de la mezcla.

Por otro lado, para los diseños propuestos se asumió una densidad de $2000 \mathrm{~kg} / \mathrm{m}^{3}$ la cual está dentro de los parámetros de densidades del concreto poroso, según la ACI 522R-06 [6], y el mismo permite el aumento de los parámetros de diseño (cemento, grava, agua).

Para cada una de las seis series mencionadas anteriormente se fabricaron cuatro cilindros cuyas medidas se especifican en la norma ASTM C-39 [8], cuyo ensayo se realizaba a los 7 y 28 días. Adicional se fabricó una losa de 16"x16"x3" la misma se utilizó para evaluar la permeabilidad.

A continuación, se muestra la tabla 2 donde se aprecia el resumen del diseño para gravas de diámetro de 3/4" con una relación de agregado/cemento 1:4 y la Tabla 3 donde se muestra el resumen del diseño para gravas de diámetro de 1/2" y $3 / 8$ " con una relación de agregado/cemento 1:4.5.

Tabla 2. Resumen de diseño del concreto poroso donde predomine la grava $3 / 4$ "

\begin{tabular}{|c|c|}
\hline \multicolumn{2}{|c|}{ Diseño donde predomine la grava 3/4" } \\
\hline \multicolumn{2}{|c|}{ Proporciones para el diseño } \\
\hline A/C & 0.36 \\
\hline Aditivo $(\%)$ & $0.30 \%$ \\
\hline Cemento $(\mathrm{kg})$ & 26.43 \\
\hline Agregado Grueso $(\mathrm{kg})$ & 105.71 \\
\hline Agua $(\mathrm{kg})$ & 9.51 \\
\hline
\end{tabular}

Tabla 3. Resumen de diseño del concreto poroso para gravas de $1 / 2$ " y $3 / 8$ "

\begin{tabular}{|c|c|}
\hline \multicolumn{2}{|c|}{ Diseño donde predomine la grava 1/2”' y 3/8” } \\
\hline \multicolumn{2}{|c|}{ Proporciones para el Diseño } \\
\hline A/C & 0.36 \\
\hline Aditivo (\%) & $0.30 \%$ \\
\hline Cemento $(\mathrm{kg})$ & 24.17 \\
\hline Agregado Grueso $(\mathrm{kg})$ & 108.78 \\
\hline Agua $(\mathrm{kg})$ & 8.70 \\
\hline
\end{tabular}




\section{Resultados}

A continuación, se muestran los resultados obtenidos dentro de los ensayos y posteriormente el análisis que se ha realizado para el presente estudio.

\subsection{Revenimiento}

A continuación, en la tabla 4 se muestra los resultados obtenidos en el revenimiento.

Tabla 4. Resultados de las series para la prueba de revenimiento

\begin{tabular}{|c|c|}
\hline $\begin{array}{c}\text { Concreto poroso } \\
\text { (Nominación de Grava) }\end{array}$ & $\begin{array}{c}\text { Revenimiento } \\
\text { (pulgadas) }\end{array}$ \\
\hline Agregado 3/4" & 0 \\
\hline Agregado 1/2" & 0.2 \\
\hline Agregado 3/8" & 0.2 \\
\hline $\begin{array}{c}\text { Agregado combinado 75\% de 3/4" y } \\
\text { 25\% de 3/8" }\end{array}$ & 0.1 \\
\hline $\begin{array}{c}\text { Agregado combinado 75\% de 1/2" y } \\
\text { 25\% de 3/8" }\end{array}$ & 0.3 \\
\hline Agregado premezclado & 0.2 \\
\hline
\end{tabular}

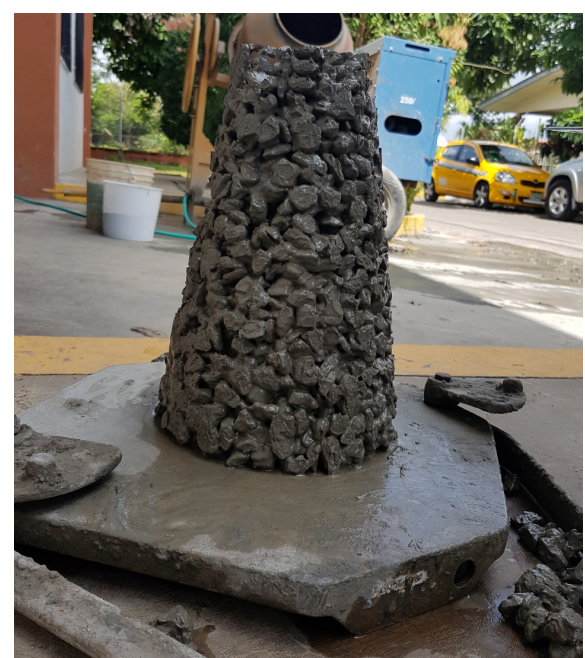

Figura 8. Prueba de revenimiento a la serie 1 , con agregado de $3 / 4 "$.

Como se puede observar en la tabla 4, el revenimiento para todas las series es casi nula. La serie 1 de material $3 / 4$ " presentó el menor revenimiento que fue de 0 mientras que la mayor la presentó la serie 5 de material combinado de $1 / 2$ " con $3 / 8$ " con un revenimiento de 0.3 $\mathrm{cm}$.

A pesar que se usó el aditivo en las seis series, no afectó directamente al ensayo de revenimiento en cuanto a su consistencia, como se muestra en la figura 8 , sin embargo sí ayudó a su trabajabilidad y retardo del fraguado gracias a la reducción de su uso al $0.3 \%$.

\subsection{Resistencia a Compresión}

Los resultados al ensayo de la resistencia a compresión del concreto poroso se muestran en la tabla 5 y tabla 6 a continuación.

Tabla 5. Resultados al ensayo a compresión de las series con grava de una sola nominación

\begin{tabular}{|c|c|c|c|}
\hline \multicolumn{4}{|c|}{ Resistencia a compresión Serie 1 (3/4") } \\
\hline & & Esfuerzo & \\
\hline Día & Carga (kg) & $\mathrm{kg} / \mathrm{cm}^{2}$ & $\begin{array}{c}\text { Promedio } \\
\left(\mathrm{kg} / \mathrm{cm}^{2}\right)\end{array}$ \\
\hline \multirow{2}{*}{28} & 23400 & 132.42 & \multirow{2}{*}{135.25} \\
\hline & 24400 & 138.08 & \\
\hline \multicolumn{4}{|c|}{ Resistencia a compresión Serie 2 (1/2") } \\
\hline & & Esfuerzo & \\
\hline Día & Carga $(\mathbf{k g})$ & $\mathbf{k g} / \mathrm{cm}^{2}$ & $\begin{array}{c}\text { Promedio } \\
\left(\mathrm{kg} / \mathrm{cm}^{2}\right)\end{array}$ \\
\hline \multirow{2}{*}{28} & 29800 & 168.63 & \multirow{2}{*}{160.71} \\
\hline & 27000 & 152.79 & \\
\hline \multicolumn{4}{|c|}{ Resistencia a compresión Serie 3 (3/8”) } \\
\hline & & Esfuerzo & \\
\hline Día & Carga (kg) & $\mathrm{kg} / \mathrm{cm}^{2}$ & $\begin{array}{c}\text { Promedio } \\
\left(\mathrm{kg} / \mathrm{cm}^{2}\right)\end{array}$ \\
\hline \multirow{2}{*}{28} & 35600 & 201.45 & \multirow{2}{*}{197.49} \\
\hline & 34200 & 193.53 & \\
\hline
\end{tabular}

Tabla 6. Resultados al ensayo a compresión de las series con grava combinada

\begin{tabular}{|c|c|c|c|}
\hline \multicolumn{4}{|c|}{$\begin{array}{c}\text { Resistencia a compresión Serie } 4 \\
(75 \% \text { 3/4"- } 25 \% \text { 3/8”) }\end{array}$} \\
\hline & & Esfuerzo & \\
\hline Día & Carga (kg) & $\mathrm{kg} / \mathrm{cm}^{2}$ & $\begin{array}{c}\text { Promedio } \\
\left(\mathrm{kg} / \mathrm{cm}^{2}\right)\end{array}$ \\
\hline \multirow{2}{*}{28} & 27000 & 152.79 & \multirow{2}{*}{149.96} \\
\hline & 26000 & 147.13 & \\
\hline \multicolumn{4}{|c|}{$\begin{array}{c}\text { Resistencia a compresión Serie } 5 \\
(75 \% 1 / 2 "-25 \% 3 / 8 ")\end{array}$} \\
\hline & & Esfuerzo & \\
\hline Día & Carga (kg) & $\mathrm{kg} / \mathrm{cm}^{2}$ & $\begin{array}{c}\text { Promedio } \\
\left(\mathrm{kg} / \mathrm{cm}^{2}\right)\end{array}$ \\
\hline \multirow{2}{*}{28} & 31000 & 175.42 & \multirow{2}{*}{176.55} \\
\hline & 31400 & 177.69 & \\
\hline
\end{tabular}




\begin{tabular}{|c|c|c|c|}
\hline \multicolumn{4}{|c|}{ Resistencia a compresión Serie 6 (Premezclado) } \\
\hline \multirow{2}{*}{ Día } & Carga (kg) & Esfuerzo & \\
\hline \multirow{2}{*}{28} & 46800 & 264.83 & $\begin{array}{c}\text { Promedio } \\
\left(\mathbf{k g} / \mathbf{c m}^{2}\right)\end{array}$ \\
\cline { 2 - 4 } & 41000 & 232.01 & 248.42 \\
\hline
\end{tabular}

\subsection{Permeabilidad}

Los resultados de la prueba de permeabilidad se muestran a continuación en la tabla 7 y tabla 8 .

Tabla 7. Resultados en las pruebas de permeabilidad del concreto poroso para series 1,2 y 3

\begin{tabular}{|c|c|c|c|}
\hline Número de Serie & 1 & 2 & 3 \\
\hline Prehumectación (seg) & 12.26 & 12.26 & 13.30 \\
\hline Cantidad Vertida (kg) & 18 & 18 & 18 \\
\hline $\begin{array}{c}\text { Tiempo de Filtración } \\
\text { (seg) }\end{array}$ & 36.03 & 36.03 & 37.07 \\
\hline $\begin{array}{c}\text { Agua filtrada por } \\
\text { Losa (kg) }\end{array}$ & 17.75 & 17.75 & 17.35 \\
\hline $\begin{array}{c}\text { Agua Retenida por } \\
\text { Losa (lt) }\end{array}$ & 0.25 & 0.25 & 0.65 \\
\hline $\begin{array}{c}\text { Infiltración } \\
\text { (pulgada/hora) }\end{array}$ & 1065.02 & 1065.02 & 1035.14 \\
\hline Infiltración (mm/hr) & 27051.5 & 27051.5 & 26292.5 \\
\hline
\end{tabular}

Tabla 8. Resultados en las pruebas de permeabilidad del concreto poroso para series 4,5 y 6

\begin{tabular}{|c|c|c|c|}
\hline Número de Serie & 4 & 5 & 6 \\
\hline Prehumectación (seg) & 13.53 & 10.79 & 13.37 \\
\hline Cantidad Vertida (kg) & 18 & 18 & 18 \\
\hline $\begin{array}{c}\text { Tiempo de Filtración } \\
\text { (seg) }\end{array}$ & 35.53 & 35.21 & 37.90 \\
\hline $\begin{array}{c}\text { Agua filtrada por } \\
\text { Losa (kg) }\end{array}$ & 17.85 & 17.85 & 17.65 \\
\hline $\begin{array}{c}\text { Agua Retenida por } \\
\text { Losa (lt) }\end{array}$ & 0.15 & 0.15 & 0.35 \\
\hline $\begin{array}{c}\text { Infiltración } \\
\text { (pulgada/hora) }\end{array}$ & 1050.53 & 1041.07 & 1012.47 \\
\hline Infiltración (mm/hr) & 26683.5 & 26443.2 & 25716.7 \\
\hline
\end{tabular}

\section{Análisis De Resultados}

5.1 Análisis de Resultados en los ensayos de resistencia a compresión

En los ensayos a compresión, las series donde se presentó una sola nominación de grava, es decir las series
1,2 y 3 , ver tabla 5; se pudo observar que la resistencia más baja fue de la serie 1 de $3 / 4$ ", esto se debe a que la partícula es más grande y a pesar que tenga el mismo volumen para un cilindro, no tiene la misma capacidad para soportar carga.

Para la serie 2 de 1/2" presentó un ligero aumento en la resistencia a los 28 días, como era de esperarse ya que la partícula al ser de menor tamaño que 3/4" aumenta la cohesión entre las partículas, mejorando de esta manera el confinamiento entre ellas. El aumento presentado fue de $18.8 \%$ de la resistencia respecto a la serie 1 .

Para la serie 3 de $3 / 8$ " se presentaron los mejores resultados para las series de una sola nominación, donde aumentó un $46 \%$ más de resistencia con respecto a la serie 1. En la tabla 9 se tabulan los resultados con el porcentaje en aumento de resistencia a medida que se disminuye el tamaño nominal de la grava.

Tabla 9. Aumento de resistencias en las series de una sola nominación

\begin{tabular}{|c|c|c|}
\hline $\begin{array}{c}\text { Número de } \\
\text { Serie }\end{array}$ & $\begin{array}{c}\text { Promedio de } \\
\text { resistencia a 28 } \\
\text { días }\left(\mathbf{k g} / \mathbf{c m}^{2}\right)\end{array}$ & $\begin{array}{c}\text { Aumento en } \\
\text { resistencia }\end{array}$ \\
\hline Serie 1 & 135.25 & \\
\hline Serie 2 & 160.71 & $18.8 \%$ \\
\hline Serie 3 & 193.53 & $46 \%$ \\
\hline
\end{tabular}

Por otro lado, para las series de nominación combinadas 4 y 5 presentaron ligeros aumentos en resistencia cuando se combinaba con el $25 \%$ de agregado $3 / 8$ ". Para la serie 1 de $3 / 4$ " y la serie 4 de $3 / 4$ " con 3/8" se presentó un aumento del $10.8 \%$ de la resistencia gracias al porcentaje de $3 / 8$ " que presentaba la serie 4 , ver tabla 10 .

Tabla 10. Aumento de resistencia en la serie 4 con respecto a la serie 1 .

\begin{tabular}{|c|c|c|}
\hline $\begin{array}{c}\text { Número de } \\
\text { Serie }\end{array}$ & $\begin{array}{c}\text { Promedio de } \\
\text { resistencia a 28 días } \\
\left(\mathbf{k g} / \mathbf{c m}^{2}\right)\end{array}$ & $\begin{array}{c}\text { Aumento en } \\
\text { Resistencia }\end{array}$ \\
\hline Serie 1 & 135.25 & \\
\hline Serie 4 & 149.96 & $\mathbf{1 0 . 8 \%}$ \\
\hline
\end{tabular}

El 25\% de agregado 3/8" que tiene la serie 4 contribuye en los espacios de mayor tamaño en la estructura del concreto poroso sean rellenados y de esta 
manera tener más confinamiento entre las partículas junto con la pasta de cemento para tener ese aumento en la resistencia.

De igual manera para la serie 5 de $1 / 2$ " con $3 / 8$ " se presentó un aumento de la resistencia con el $25 \%$ de agregado de 3/8" con respecto a la serie 2 de 1/2". El aumento presentado fue de un $10 \%$ con respecto a la serie 2, ver Tabla 11.

Tabla 11. Aumento de resistencia en la serie 5 con respecto a la serie 2

\begin{tabular}{|c|c|c|}
\hline Número de Serie & $\begin{array}{c}\text { Promedio de } \\
\text { resistencia a 28 días } \\
\left(\mathbf{k g} / \mathbf{c m}^{2}\right)\end{array}$ & $\begin{array}{c}\text { Aumento en } \\
\text { resistencia }\end{array}$ \\
\hline Serie 2 & 160.71 & \\
\hline Serie 5 & 176.55 & $\mathbf{1 0 \%}$ \\
\hline
\end{tabular}

Para la serie número 6 de premezclado en el cual no se hace ningún tipo de división de material o porcentajes, presentó los mejores resultados de resistencia en todas las series, gracias a la combinación de los tres tipos de gravas que se utilizaron en el concreto. El confinamiento entre las partículas fue el mejor, de manera que cuando estaban sometidos a cargas se lograra alcanzar resistencias más elevadas.

Por medio de la granulometría del agregado se puede observar qué porcentaje de cada nominación de material se encontraba en el concreto de la serie 6. Los resultados del porcentaje de cada material se muestran en la tabla 12.

Tabla 12. Porcentaje de cada grava en serie 6

\begin{tabular}{|c|c|}
\hline Tipo de grava & \% que contenía \\
\hline $3 / 4 "$ & $61.64 \%$ \\
\hline $1 / 2 "$ & $32.15 \%$ \\
\hline $3 / 8 "$ & $5.47 \%$ \\
\hline $\mathrm{N} .{ }^{\circ} 4$ & $0.09 \%$ \\
\hline $\mathrm{N} .{ }^{\circ} 8$ & $0.65 \%$ \\
\hline
\end{tabular}

Como se puede observar en la tabla 12, la grava predominante en la serie era la de $3 / 4$ ". Al tener mayor porcentaje de esta grava, hay mayor cantidad de vacíos en la estructura del concreto. La grava de 1/2" ayudaba llenar estos huecos dejados por el material 3/4" y de esta manera mejorar el confinado. Lo mismo pasaba con la grava 3/8" que a pesar de tener un bajo porcentaje en el concreto, ayudaba a tener más confinamiento en la estructura del concreto. Los demás porcentajes eran muy bajos por los que se podían considerar despreciables para una amasada.

De esta manera, en resumen, los mejores resultados para las series de una sola nominación la presentó la serie 3 de material único nominal de $3 / 8$ ", mientras que los mejores resultados para las series combinadas la presentó la serie 6 de material premezclado. En la tabla 13 se puede ver los resultados para estas dos series.

Tabla 13. Mejores resultados presentados en los ensayos a compresión

\begin{tabular}{|c|c|}
\hline Número de Serie & $\begin{array}{c}\text { Promedio de resistencia a } \\
\mathbf{2 8} \text { días }\left(\mathbf{k g} / \mathbf{c m}^{\mathbf{2}}\right)\end{array}$ \\
\hline Serie 3 & 193.53 \\
\hline Serie 6 & 248.42 \\
\hline
\end{tabular}

\subsection{Tipos de fallas presentadas}

Según la norma ASTM C39 [8] hay seis tipos de fallas que depende de muchos factores como lo son: la formación de los conos, confinamiento de las partículas, nivelación de los cabezales, entre otros. En la figura 9 se muestran los tipos de fallas que se presentaron en los ensayos.

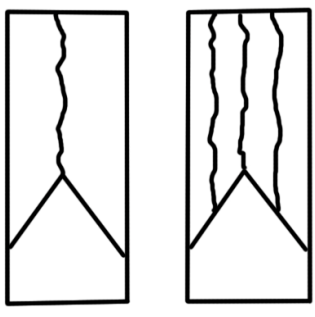

Tipo 2

Cono bien formado en un extremo, grietas verticales que atraviesan las tapas, sin cono bien definido en

el otro extremo.

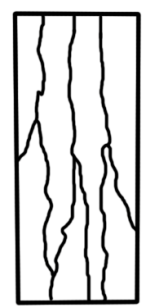

Tipo 3

Fisuras verticales a través de cada extremo, sin conos bien formados.
Figura 9. Esquema de los patrones típicos de fractura (ASTM C39) [8].

Al realizar los ensayos a compresión a los especímenes de concreto poroso, se obtuvieron los 
siguientes tipos de fallas que se describen en la tabla 14 y tabla 15.

Tabla 14. Tipos de fallas presentadas en el concreto poroso con grava de una sola nominación

\begin{tabular}{|c|c|c|c|}
\hline $\begin{array}{l}\text { Número } \\
\text { de Serie }\end{array}$ & $\begin{array}{l}\text { Tipo de } \\
\text { material }\end{array}$ & $\begin{array}{l}\text { Edad } \\
\text { (Días) }\end{array}$ & $\begin{array}{c}\text { Tipo de } \\
\text { falla } \\
\end{array}$ \\
\hline \multirow{4}{*}{1} & \multirow{4}{*}{$3 / 4 "$} & \multirow{2}{*}{7} & 3 \\
\hline & & & 2 \\
\hline & & \multirow{2}{*}{28} & 3 \\
\hline & & & 3 \\
\hline \multirow{4}{*}{2} & \multirow{4}{*}{$1 / 2 "$} & \multirow{2}{*}{7} & 3 \\
\hline & & & 2 \\
\hline & & \multirow{2}{*}{28} & 3 \\
\hline & & & 3 \\
\hline \multirow{4}{*}{3} & \multirow{4}{*}{$3 / 8 "$} & \multirow{2}{*}{7} & 3 \\
\hline & & & 3 \\
\hline & & \multirow{2}{*}{28} & 3 \\
\hline & & & 3 \\
\hline
\end{tabular}

Tabla 15. Tipos de fallas presentadas en el concreto poroso con grava combinada

\begin{tabular}{|c|c|c|c|}
\hline $\begin{array}{l}\text { Número de } \\
\text { Serie }\end{array}$ & $\begin{array}{l}\text { Tipo de } \\
\text { material }\end{array}$ & $\begin{array}{l}\text { Edad } \\
\text { (Días) }\end{array}$ & $\begin{array}{l}\text { Tipo de } \\
\text { falla }\end{array}$ \\
\hline \multirow{4}{*}{4} & \multirow{4}{*}{$\begin{array}{l}75 \%-3 / 4 " \text { " } \\
25 \%-3 / 8 ”\end{array}$} & \multirow{2}{*}{7} & 3 \\
\hline & & & 2 \\
\hline & & \multirow{2}{*}{28} & 3 \\
\hline & & & 3 \\
\hline \multirow{4}{*}{5} & \multirow{4}{*}{$\begin{array}{l}75 \%-1 / 2 ” \text { y } \\
25 \%-3 / 8 ”\end{array}$} & \multirow{2}{*}{7} & 2 \\
\hline & & & 3 \\
\hline & & \multirow{2}{*}{28} & 3 \\
\hline & & & 3 \\
\hline \multirow{4}{*}{6} & \multirow{4}{*}{ Premezclado } & \multirow{2}{*}{7} & 3 \\
\hline & & & 3 \\
\hline & & \multirow{2}{*}{28} & 3 \\
\hline & & & 3 \\
\hline
\end{tabular}

En los resultados se puede observar que la falla predominante es la falla tipo 3, la que corresponde a un agrietado vertical. Esto se debe a la uniformidad de los agregados en el molde del cilindro, al no tener agregado fino el cilindro depende directamente del agregado y la pasta de cemento, lo que dificulta el varillado y la formación del cilindro. Lo mismo pasa con la falla tipo 2 , que produce grietas verticales en el cilindro ya que los conos no están bien formados por la uniformidad de los agregados. Garantizar la homogeneidad en el concreto poroso a la hora de fabricar un espécimen se dificulta a medida de que el tamaño nominal es más grande. En la figura 10 se muestra las fallas tipos 2 presentadas en el concreto poroso y en la figura 11 las fallas tipo 3 .

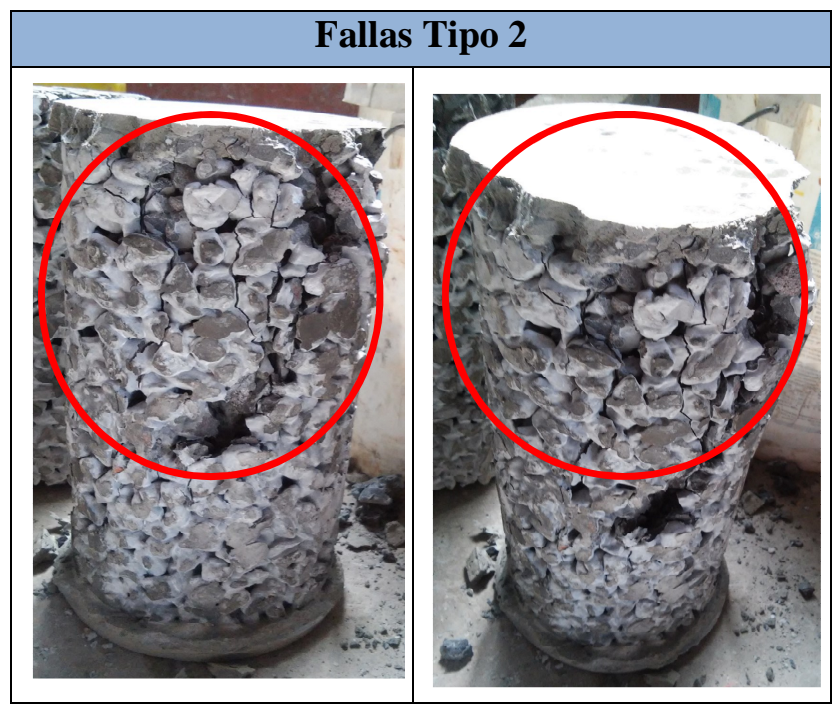

Figura 10. Fallas Tipo 2 presentadas en la serie 1 de agregado $3 / 4$ ".

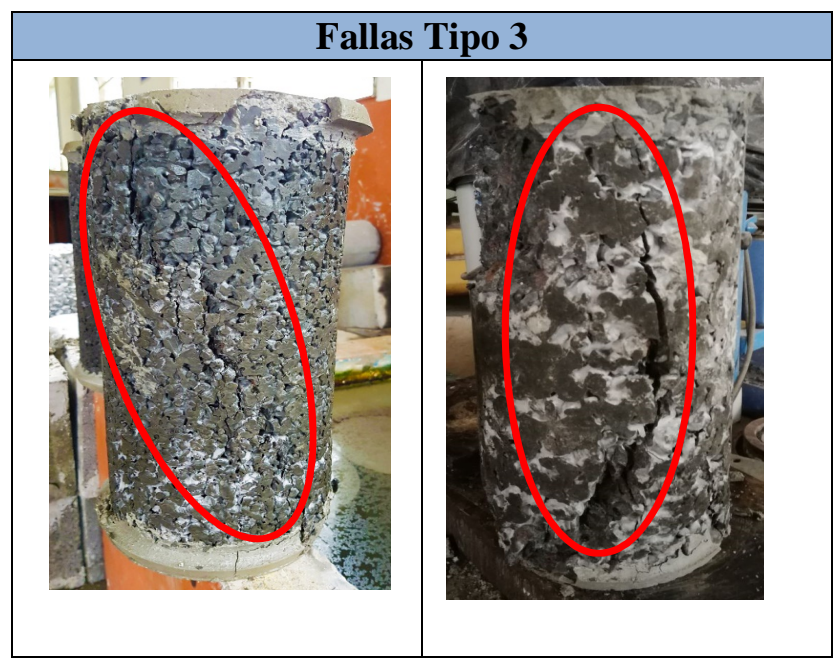

Figura 11. Fallas Tipo 3 presentadas en la serie 3 de agregado $3 / 8$ " (izquierda) y en el premezclado (derecha).

\subsection{Análisis de resultados en los ensayos de permeabilidad}

Las variaciones en la tasa de filtración fueron mínimas en cada serie. En la tabla 18 se muestran los resultados de las tasas de filtración para cada serie en unidades de pulgadas/hora. 
Tabla 16. Resultados de la permeabilidad de las series en unidades (pulgadas/hora)

\begin{tabular}{|c|c|}
\hline Número de serie & $\begin{array}{c}\text { Tasa de filtración } \\
\text { (pulgadas/hora) }\end{array}$ \\
\hline Serie 1 & 1065.02 \\
\hline Serie 2 & 1065.02 \\
\hline Serie 3 & 1035.14 \\
\hline Serie 4 & 1050.53 \\
\hline Serie 5 & 1041.07 \\
\hline Serie 6 & 1012.47 \\
\hline
\end{tabular}

Como se observa en la tabla 16 , los resultados tienen pocas variaciones entre sí. Esto se debe a que tanto los parámetros de diseños como el proceso de capas y compactación en la fabricación de las losas fueron los mismos en todos. Se alcanzó a consolidar los diferentes tipos de gravas puestas en estudio con la pasta de cemento para que de esta manera el porcentaje de vacíos fuera el mismo en todas las series. De esta manera alcanzando resultados óptimos para todos los ensayos a compresión y permeabilidad.

\section{Conclusiones}

De acuerdo a la investigación realizada y subsecuente al análisis de los resultados obtenidos, se pueden establecer las siguientes conclusiones:

1. Los resultados de las series indican que, utilizando una relación de agua/cemento de 0.36 se consigue una mejor manejabilidad y trabajabilidad del concreto poroso y de esta manera garantizar el confinamiento y adherencia de la grava con la pasta de cemento.

2. Los resultados muestran que a medida que disminuye el tamaño nominal de la grava aumenta la resistencia a compresión debido al confinamiento de las partículas. Mientras que para la permeabilidad a medida que el tamaño de la grava aumenta, mayor será la permeabilidad.
3. La nominación de grava de $3 / 8$ " fue la que más aportó en las resistencias a la hora de combinarse con otro tipo de grava.

4. La capacidad de filtración del concreto poroso no depende únicamente de la granulometría del agregado, esta se ve afectada, por la relación agua/cemento y la homogeneidad que se logre al momento de la fabricación, es importante procurar que los poros se encuentren conectados entre sí, de manera que el paso del agua a través de la superficie porosa sea continua.

5. El concreto poroso formado con grava de $3 / 8$ " y de material premezclado de $3 / 4$ " presentaron resultados a compresión por encima de lo estipulado en la Norma ACI 522R-06 [6], al igual que en permeabilidad. Por lo que se recomienda el uso de este concreto con estos tipos de grava en las aplicaciones en campo.

\section{Referencias}

[1]. Carlos Aire (2008), "El hormigón permeable como alternativa sostenible".

[2]. Ligia M. Vélez (2010), "Permeabilidad y Porosidad del Concreto".

[3]. Juan Orlando López Orozco (2004), "Porosidad del Concreto".

[4]. Barahona, R. A. , Martinez, M. V. , Zelaya, S. E. (2013), "Comportamiento Del Concreto Permeable Utilizando Agregado Grueso De Las Canteras, El Carmen, Aramuaca Y La Pedrera, De La Zona Oriental De El Salvador".

[5]. Paul D. Tennis, Michael L. Leming, and David J. Akers (2004) "Pervious Concrete Pavements".

[6]. 522R-06 Pervious Concrete (2006) - ACI Committee 522.

[7]. Standard Test Method for Slump of Hydraulic-Cement Concrete - ASTM C143 (2015).

[8]. Standard Test Method for Compressive Strength of Cylindrical Concrete Specimens - ASTM C39 (2014).

[9]. Standard Test Method for Infiltration Rate of In Place Pervious Concrete - ASTM C1701 (2017). 